\title{
Capacidad cardio-respiratoria de niños escolares que viven a moderada altitud
}

\author{
ROSSANA GÓMEZ-CAMPOS ${ }^{1,2}$, MIGUEL ARRUDA ${ }^{1}$, \\ ALEJANDRO ALMONACID-FIERRO ${ }^{2}$, EDÍLSON HOLBOLD ${ }^{1}$, \\ CRISTIANE AMARAL-CAMARGO ${ }^{3}$, DIEGO GAMERO ${ }^{1}$, MARCO A. COSSIO-BOLANOS ${ }^{4,5}$ \\ 1. Facultad de Educación Física de la Universidad Estadual de Campinas, Sao Paulo, Brasil. \\ 2. Universidad Autónoma de Chile, Chile. \\ 3. Facultad de Ciencias Médicas de la Universidad Estadual de Campinas, Sao Paulo, Brasil. \\ 4. Departamento Ciencias de la Actividad Física. Universidad Católica del Maule, Chile. \\ 5. Instituto del Deporte Universitario, Universidad Nacional San Agustín, Arequipa, Perú.
}

\section{ABSTRACT \\ Cardiorespiratory capacity in children living at moderate altitude}

Objective: To determine the cardiorespiratory capacity of school children living at moderate altitude. Patients and Method: 795 children (394 children and 401 girls) were selected from urban public schools in Arequipa, Peru at moderate altitude $(2,320 \mathrm{~m})$. Anthropometric variables (body mass, height, body fat percentage) and cardiorespiratory capacity were assessed using the Course Navette test, considering the following categories: deficient, poor, fair, good, very good and excellent. Results: The results showed significant differences in all categories $(\mathrm{p}<0.05)$. It was described that the median values of the poor and deficient categories in both gender were lower than what is considered acceptable $(\mathrm{p}<0.05)$. It was concluded that $19 \%$ of boys and $21 \%$ of girls showed low level of cardiorespiratory capacity and a low negative correlation with overweight $(\mathrm{r}=$ -0.20 to -0.22$)$ and a moderate negative correlation with obesity $(r=-0.39$ to -0.42$)$ were described for both genders. Conclusions: Low levels of cardiorespiratory capacity in boys and girls living at moderate altitude are observed, which is negatively correlated with excess body weight. The results suggest that 1 in 5 children are likely to suffer some type of cardiovascular event.

(Key words: Cardiorespiratory capacity, kids, school children, moderate altitude).

Rev Chil Pediatr 2014; 85 (2): 188-196

\section{RESUMEN}

Objetivo: Determinar la capacidad cardio-respiratoria de niños escolares que viven a moderada altitud. Pacientes y Método: Fueron seleccionados 795 niños (394 niños y 401 niñas) de escuelas públicas del área urbana de moderada altitud $(2.320 \mathrm{msnm})$ de Arequipa, Perú. Se evaluaron variables antropométricas (masa corporal, estatura, porcentaje de grasa) y capacidad cardio-respiratoria a través del test indirecto de Course

Recibido el 5 de junio de 2013, devuelto para corregir el 14 de octubre de 2013, segunda versión 23 de octubre de 2013 , aceptado para publicación el 28 de noviembre de 2013.

Este trabajo cumple con los requisitos sobre consentimiento /asentimiento informado, comité de ética, financiamiento, estudios animales y sobre la ausencia de conflictos de intereses según corresponda.

Correspondencia a:

Rossana Gómez-Campos

E-mail: rossanagomez_c@hotmail.com 
Navette, considerando las siguientes categorías: deficiente, malo, aceptable, bueno, muy bueno y excelente. Resultados: Los resultados muestran diferencias significativas en todas las categorías $(p<0,05)$. Se verificó que los valores medios de las categorías malo y deficiente en ambos sexos son inferiores a los de la categoría aceptable $(\mathrm{p}<0,05)$. Se determinó que el $19 \%$ de niños y $21 \%$ de niñas mostraron bajo nivel de capacidad cardio-respiratoria, además se verificó correlación negativa baja con la categoría sobrepeso $(\mathrm{r}=-0,20$ a $-0,22)$ y moderada con la obesidad $(r=-0,39$ a $-0,42)$ en ambos sexos. Conclusiones: Existen bajos niveles de capacidad cardio-respiratoria en niños de ambos sexos niños escolares que viven a moderada altitud, que se correlaciona negativamente con el exceso de peso corporal. Los resultados sugieren que uno de cada 5 niños se encuentra con posibilidades de padecer algún evento de índole cardiovascular.

(Palabras clave: Capacidad cardio-respiratoria, niños, escolares, moderada altitud).

Rev Chil Pediatr 2014; 85 (2): 188-196

\section{Introducción}

El término de condición física ha ido evolucionando a lo largo del tiempo, pasando de una orientación tradicional vinculada al rendimiento deportivo hacia una orientación mucho más cercana y relacionada con la salud ${ }^{1}$. Este término, es definido como la capacidad de llevar a cabo las tareas diarias con vigor y vivacidad sin excesiva fatiga y con suficiente energía para disfrutar del tiempo libre u ocio y para afrontar emergencias inesperadas ${ }^{2}$. Expresa una estrecha relación en la cual, el nivel de condición física está afectado por la cantidad y tipo de actividad física realizada habitualmente. Para su valoración, se considera la participación de componentes asociados a las dimensiones morfológicas, funcional-motoras, fisiológicas y comportamentales ${ }^{3}$.

La dimensión funcional-motora, incluye datos relacionados a la función cardio-respiratoria y músculo-esquelética, dentro de tales componentes la resistencia cardio-respiratoria ha sido considerada una de las más importantes, tanto para la gran mayoría de los atletas de las diferentes modalidades deportivas, como para los individuos no atletas que necesitan de la práctica de la actividad física moderada como medio de promoción de la salud ${ }^{4}$. Inclusive dicha práctica se debe extender a las poblaciones escolares con el propósito de promover y desarrollar estilos de vida saludables a futuro.

En general, se entiende por resistencia cardio-respiratoria la capacidad para realizar tareas moderadas que impliquen la participación de grandes masas musculares durante períodos prolongados de tiempo. Los factores como la edad, sexo, tipo de fibra, naturaleza del ejercicio, adaptaciones al entrenamiento, factores psicológicos y el ambiente pueden influir en la capacidad de la actividad muscular aeróbi$\mathrm{ca}^{5}$. De hecho, el desarrollo de la resistencia cardio-respiratoria en niños, adolescentes y jóvenes se asocia a la disminución de la prevalencia de enfermedades cardiovasculares en niños y adolescentes.

Desde esa perspectiva, los bajos índices de participación en los programas de actividad física y deportes en niños, jóvenes y adultos son motivo de preocupación permanente en la sociedad, dado que la prevalencia del exceso de peso y la obesidad se han incrementado de forma significativa ${ }^{6}$, constituyendo un grave problema de salud pública, tanto en países desarrollados, como en aquellos en desarrollo, afectando a niños, adolescentes y adultos ${ }^{7}$.

En este sentido, es necesario considerar los beneficios que proporciona la actividad física sobre la capacidad cardio-respiratoria, sobre todo, si se practica de forma regular y sistemática a edades cada vez más tempranas, lo que podría contribuir en la adopción de estilos de vida más saludables durante todo el ciclo de la vida. En el Perú los programas escolarizados dependen de las directrices curriculares emanadas por el Ministerio de Educación, a través del cual se promueven programas de actividad física-deportiva a nivel inicial, primaria y secundaria; sin embargo, tales programas son desarrollados una vez por semana, lo que poco o nada podría contribuir en la adquisición de 
aceptables niveles de capacidad cardio-respiratoria en escolares peruanos.

Por ello, es necesario contar con estudios descriptivos de referencia para poder caracterizar a los escolares que viven en diferentes condiciones geográficas incluyendo a aquellos que viven a moderada altitud respecto a la capacidad cardio-respiratoria. El objetivo del estudio fue determinar la capacidad cardio-respiratoria de niños escolares que viven a moderada altitud.

\section{Pacientes y Método}

\section{Tipo de estudio}

El estudio fue de tipo descriptivo de corte transversal.

\section{Selección de la muestra}

El universo estuvo conformado por un total de 5.627 alumnos, siendo 2.790 hombres y 2.837 mujeres de condición socioeconómica media. El tamaño óptimo de la muestra se determinó de forma probabilística estratificada $(\mathrm{IC}=95 \%)$, determinándose 795 niños de ambos sexos, siendo 394 niños y 401 niñas de edad escolar pertenecientes al sistema educativo Peruano de educación básica regular. Los escolares pertenecían a cuatro escuelas públicas del área urbana de Arequipa, Perú, localizada a moderada altitud (2.320 msnm). Los niños realizaban sesiones de educación física de 80-90 $\mathrm{min} /$ día (una vez por semana). El rango de edad para ambos géneros comprende: $6,0-6,9,7,0-7,9,8,0-8,9,9,0-9,9,10,0-10,9$, $11,0-11,9$ años. Se incluyeron en el estudio a todos los niños cuyos padres y/o apoderados autorizaron la participación en el estudio. Los niños que realizaban actividad física superior a 2-3 sesiones durante la semana fueron excluidos del estudio, dado que podrían evidenciar mejores niveles de capacidad cardiovascular, debido a la realización de mayor frecuencia de actividad física durante una semana.

Todos los padres y/o apoderados fueron informados sobre el estudio, a partir del cual, firmaron la ficha de consentimiento informado, autorizando la valoración de medidas antropométricas y del test de capacidad cardio- respiratoria, de acuerdo a las recomendaciones del Comité de Ética del Departamento Médico del Instituto del Deporte Universitarios IDUNSA de la Universidad Nacional de San Agustín de Arequipa.

Técnicas e instrumentos. Para la evaluación de las variables antropométricas se adoptó el protocolo estandarizado por la "International Working Group of Kineanthropometry" descrita por Ross y Marfell-Jones ${ }^{8}$, porque ofrecen mayor grado de confiabilidad para las variables antropométricas y por ser utilizada en estudios de crecimiento y desarrollo.

Todas las variables fueron evaluadas con la menor cantidad de ropa posible y los pliegues cutáneos tricipital y subescapular fueron medidas en el lado derecho del cuerpo.

Para determinar la masa corporal $(\mathrm{kg})$, se evaluó descalzo y con la menor cantidad de ropa posible, utilizando una balanza digital con una precisión de $(200 \mathrm{~g})$ de marca Tanita con una escala de (0 a $150 \mathrm{~kg})$.

Para determinar la estatura $(\mathrm{cm})$, se evaluó a los sujetos ubicado en el plano de Frankfurt (meatoorbitario) sin zapatos, utilizando un estadiómetro de aluminio de marca seca graduada en milímetros, presentando una escala de $(0-2,5 \mathrm{~m})$.

Los valores de los pliegues cutáneos ( $\mathrm{mm})$, fueron evaluación en la región tricipital y subescapular de acuerdo a la línea de clivaje utilizando un compás de pliegues cutáneos Harpenden que ejerce una presión constante de $\left(10 \mathrm{~g} / \mathrm{mm}^{2}\right)$. Estos valores permitieron la determinación del porcentaje de grasa corporal $\% \mathrm{G}$ (cantidad de tejido adiposo subcutáneo) a través del uso de la ecuación de regresión propuesta por Boileau, Lohman y Slaughter':

Donde,

Hombres $\% \mathrm{G}=1,35\left(\sum T R+S E\right)-0,012\left(\sum T R+S E\right)^{2}-4,4$ y para Mujeres $\% \mathrm{G}=1,35\left(\sum T R+S E\right)-0,012\left(\sum T R+S E\right)^{2}-2,4$ donde TR significa pliegue y $S E$ pliegue subescapular.

Para analizar el estado nutricional, se utilizó el criterio del índice nutricional descrito por Hernández-Rodrigues ${ }^{10}$. Este índice se basa en la comparación de la relación simple del peso y la estatura del sujeto. Consideramos los puntos de corte por edad y sexo con relación al 
percentil 50 referidos en las tablas referenciales de la Organización Mundial de la Salud ${ }^{11}$. El valor calculado del índice permite diferenciar 4 situaciones: Inferior a $90 \%=$ bajo peso (delgadez), entre $90-110 \%=$ peso normal, entre $110-120 \%=$ sobrepeso y mayor a $120 \%=$ obesidad. Su fórmula es:

$$
I N=\frac{\operatorname{Peso}(\mathrm{kg}) / \text { Estatura }(\mathrm{m})}{\operatorname{Peso}(p 50) / \text { Estatura }(p \text { 50) }} \times 100
$$

Para la evaluación de la capacidad aeróbica se utilizó el test de Course Navette (método indirecto) que se caracteriza por ser un test de potencia aeróbica que evalúa la capacidad cardio-respiratoria. Dicho test, se realizó mediante una carrera de ida $(20 \mathrm{~m})$ y vuelta $(20 \mathrm{~m})$ en la que el sujeto va desplazándose de un punto a otro, realizando un cambio de sentido al ritmo indicado por una señal sonora (CD ROM con ajuste de señal auditiva "bip") que va acelerándose progresivamente, siguiendo las recomendaciones de Leger et $\mathrm{al}^{12}$. El test se inicia a una velocidad de $8 \mathrm{~km} / \mathrm{h}$, siendo al principio lento, incrementándose la velocidad paulatinamente con el transcurso del tiempo. El test culmina a una velocidad de $18 \mathrm{~km} / \mathrm{h}$. Durante el test, el mismo sujeto es el encargado de determinar el propio ritmo, de tal manera que se encuentre en un extremo de la pista al oír la señal, con una aproximación de 1 ó $2 \mathrm{~m}$. En cada extremo hay que tocar la línea con el pie y el test acaba en el momento en que el individuo interrumpe voluntariamente la prueba, y/o cuando no soporta más el ritmo impuesto por la señal acústica. Por lo tanto, es el momento de registrar la capacidad cardio-respiratoria. Antes de la valoración del test, todos los sujetos realizaron un calentamiento (entrada de calor) por $10 \mathrm{~min}$ seguido de ejercicios de flexibilidad.

La confiabilidad del test se verificó evaluando a través del procedimiento test y retest. E1 10\% de sujetos fueron seleccionados para realizar la prueba en dos oportunidades. El intervalo entre la primera y segunda medición fue de $48 \mathrm{~h}$. El error Técnico de Medida fue inferior al $4 \%$ y la capacidad de reproductibilidad fue de $(r=0,89-0,92)$ para ambos sexos.

\section{Criterios de referencia adoptados}

Para el diagnóstico de la capacidad cardiorespiratoria, se utilizó como referencia los puntos de corte basados en percentiles y sugeridos por Cossio-Bolaños, Arruda ${ }^{13}$ para niños que viven a moderada altitud donde consideran las siguientes categorías: $\angle \mathrm{P} 10=$ deficiente, $\mathrm{P} 10$ a P25 = Mala, P25 a P50 = Aceptable, P50 a $\mathrm{P} 75=$ Bueno, P75 a P90 = Muy bueno y $>$ P90 $=$ Excelente.

\section{Análisis estadístico}

Los resultados del estudio fueron analizados a través de la estadística descriptiva simple de media aritmética, desviación estándar y porcentajes. Para determinar las diferencias significativas entre ambos sexos se utilizó la prueba de " $t$ " Student para muestras independientes. Para determinar la diferencia entre las categorías de capacidad cardio-respiratoria en función de la edad y sexo se utilizó la prueba $\chi^{2}(p<0,05)$. Las diferencias entre las categorías se determinó por ANOVA de una vía $(p<0,05)$. Todos los datos recolectados fueron procesados por el programa Sigma Estat 4.0. En todos los casos se adoptó un nivel de significancia de $(p<0,05)$, previamente se verificó la normalidad de los datos mediante la prueba de Kolmogorov-Smirnov.

\section{Resultados}

La tabla 1 muestra los valores medios y la desviación estándar de las variables antropométricas y la capacidad cardio-respiratoria. En ambos sexos se observa valores medios crecientes en función de la edad. En la figura 1 se ilustra el aumento lineal de los metros recorridos en función de la edad, siendo similar el comportamiento aproximadamente hasta los 8 años, para luego a partir de los 9 años los niños evidencian mayor capacidad cardio-respiratoria en comparación con las niñas.

La tabla 2, muestran los valores medios y desviación estándar de seis categorías (excelente, muy bueno, bueno, aceptable, malo y deficiente) en niños de ambos sexos. Los resultados muestran diferencias significativas en todas las categorías $(\mathrm{p}<0,05)$. Se observa 
claramente que los valores medios de las categorías malo y deficiente en ambos sexos muestran valores significativamente inferiores a los de la categoría aceptable (figura 2). Además cuando se relacionó las categorías del estado nutricional (eutrófico, sobrepeso y obesidad) con los valores de la capacidad cardiovascular (metros recorridos) se observó valores negativos de Pearson que oscilan entre $(\mathrm{r}=-0,20$ a $-0,42$ ), respectivamente (figura 3 ).
Los valores de la capacidad cardio-respiratoria se observan en la tabla 3 . Los resultados muestran que el $21 \%$ de los niños y $19 \%$ de las niñas se encuentran entre las categorías de malo y deficiente, respecto a la capacidad cardio-respiratoria. Estos valores evidencian valores altamente significativos con relación a los niños que se encuentran en la categoría de aceptable condición cardio-respiratoria y/o regular (figura 2).

Tabla 1. Caracterización de la muestra estudiada

\begin{tabular}{|lccccc|}
\hline $\begin{array}{l}\text { Edad en } \\
\text { años, rango }\end{array}$ & $\mathbf{n}$ & $\begin{array}{c}\text { Peso en kg, } \\
\overline{\mathbf{x}} \pm \mathbf{D E}\end{array}$ & $\begin{array}{c}\text { Estatura en metros } \\
\overline{\mathbf{x}} \pm \mathbf{D E}\end{array}$ & $\begin{array}{c}\text { \% } \\
\text { de grasa corporal } \\
\overline{\mathbf{x}} \pm \mathbf{D E}\end{array}$ & $\begin{array}{c}\text { Capacidad Cardio-respiratoria (m) } \\
\overline{\mathbf{x}} \pm \mathbf{D E}\end{array}$ \\
Niños & & & & & \\
$6,0-7,9$ & 56 & $21,80 \pm 2,20$ & $1,14 \pm 0,05$ & $12,47 \pm 2,78$ & $266,10 \pm 66,90$ \\
$7,0-7,9$ & 67 & $22,91 \pm 3,92$ & $1,18 \pm 0,05$ & $12,67 \pm 4,04$ & $276,12 \pm 72,13$ \\
$8,0-8,9$ & 68 & $25,88 \pm 3,55$ & $1,25 \pm 0,05$ & $13,18 \pm 3,68$ & $320,88 \pm 119,70$ \\
$9,0-9,9$ & 73 & $29,38 \pm 3,80$ & $1,28 \pm 0,04$ & $14,31 \pm 4,62$ & $438,10 \pm 210,03$ \\
$10,0-10,9$ & 59 & $33,25 \pm 4,05$ & $1,36 \pm 0,07$ & $16,11 \pm 3,81$ & $468,81 \pm 216,25$ \\
$11,0-11,9$ & 71 & $37,01 \pm 4,83$ & $1,43 \pm 0,05$ & $16,93 \pm 4,32$ & $710,70 \pm 240,30$ \\
Niñas & & & & & \\
$6,0-7,9$ & 80 & $22,48 \pm 2,62$ & $1,19 \pm 0,05$ & $17,39 \pm 3,30$ & $260,25 \pm 69,17$ \\
$7,0-7,9$ & 61 & $24,62 \pm 3,45$ & $1,20 \pm 0,14$ & $17,85 \pm 4,43$ & 66,45 \\
$8,0-8,9$ & 63 & $26,57 \pm 3,04$ & $1,26 \pm 0,06$ & $20,69 \pm 2,82$ & $301,30 \pm 87,91$ \\
$9,0-9,9$ & 58 & $30,38 \pm 4,62$ & $1,32 \pm 0,06$ & $22,04 \pm 3,67$ & $381,40 \pm 132,45^{*}$ \\
$10,0-10,9$ & 63 & $37,21 \pm 5,37$ & $1,32 \pm 0,06$ & $21,33 \pm 4,60$ & $398,73 \pm 120,80^{*}$ \\
$11,0-11,9$ & 76 & $38,92 \pm 5,96$ & $1,44 \pm 0,05$ & $23,30 \pm 4,28$ & $520,53 \pm 163,66^{*}$ \\
\hline
\end{tabular}

$*(p<0,05)$ diferencia significativa en relación a los niños de la misma edad.

Tabla 2. Capacidad cardio-respiratoria $(\mathrm{m})$ de escolares de ambos sexos distribuidos por categorías

\begin{tabular}{|c|c|c|c|c|c|c|}
\hline $\begin{array}{l}\text { Edad en } \\
\text { años, rango }\end{array}$ & $\begin{array}{c}\text { Deficiente } \\
\bar{x} \pm D E\end{array}$ & $\begin{array}{c}\text { Malo } \\
\bar{x} \pm \text { DE }\end{array}$ & $\begin{array}{c}\text { Aceptable } \\
\bar{x} \pm \mathrm{DE}\end{array}$ & $\begin{array}{l}\text { Bueno } \\
\bar{x} \pm \mathrm{DE}\end{array}$ & $\begin{array}{c}\text { Muy bueno } \\
\bar{x} \pm D E\end{array}$ & $\begin{array}{c}\text { Excelente } \\
\bar{x} \pm D E\end{array}$ \\
\hline \multicolumn{7}{|l|}{ Niños } \\
\hline $6,0-7,9$ & $170,00 \pm 11,6$ & $200,0 \pm 0,0^{a}$ & $225,7 \pm 9,4^{\mathrm{ab}}$ & $268,3 \pm 10,3^{\mathrm{abc}}$ & $320,0 \pm 16,3^{\text {abcd }}$ & $368,9 \pm 10,5^{\text {abcde }}$ \\
\hline $7,0-7,9$ & $000,0 \pm 0,0$ & $200,0 \pm 0,0^{a}$ & $220,0 \pm 0,0^{a}$ & $254,4 \pm 21,7^{a b c}$ & $354,0 \pm 28,4^{\mathrm{abcd}}$ & $428,6 \pm 36,3^{\text {abcde }}$ \\
\hline $8,0-8,9$ & $166,7 \pm 10,3$ & $212 \pm 10,3^{a}$ & $254,7 \pm 9,2^{\mathrm{ab}}$ & $320,0 \pm 32,1^{\mathrm{abc}}$ & $402,7 \pm 28,2^{\mathrm{abcd}}$ & $577,1 \pm 86,7^{\text {abcde }}$ \\
\hline $9,0-9,9$ & $234,3 \pm 9,8$ & $260,0 \pm 0,0^{a}$ & $307,5 \pm 19,1^{a}$ & $402,1 \pm 37,7^{a b c}$ & $588,6 \pm 46,2^{\mathrm{abcd}}$ & $906,0 \pm 176,9^{\text {abcde }}$ \\
\hline $10,0-10,9$ & $180,0 \pm 31,0$ & $266,0 \pm 20,0^{a}$ & $366,7 \pm 37,5^{\mathrm{aB}}$ & $481,3 \pm 29,6^{\mathrm{abc}}$ & $652,0 \pm 95,8^{\text {abcd }}$ & $926,7 \pm 27,3^{\text {abcde }}$ \\
\hline $11,0-11,9$ & $360,0 \pm 0,0$ & $401,8 \pm 16,6^{a}$ & $545,7 \pm 41,8^{\mathrm{ab}}$ & $810,0 \pm 53,7^{a b c}$ & $930,9 \pm 16,4^{\mathrm{abcd}}$ & $1.003,1 \pm 7,5^{\text {abcde }}$ \\
\hline \multicolumn{7}{|l|}{ Niñas } \\
\hline $6,0-7,9$ & $160,0 \pm 0,0$ & $180,0 \pm 0,0^{a}$ & $210,8 \pm 10,2^{a}$ & $260 \pm 18,4^{\mathrm{abc}}$ & $309,2 \pm 10,4^{\mathrm{abcd}}$ & $370,8 \pm 58,1^{\text {abcde }}$ \\
\hline $7,0-7,9$ & $160,0 \pm 0,0$ & $190,3 \pm 20,7^{a}$ & $245,0 \pm 8,9^{a b}$ & $284,4 \pm 8,8^{a b c}$ & $320,0 \pm 0,0^{a b c d}$ & $372,0 \pm 10,3^{\mathrm{abcde}}$ \\
\hline $8,0-8,9$ & $170,0 \pm 11,5$ & $200,0 \pm 0,0^{\mathrm{a}}$ & $223,1 \pm 7,5^{\mathrm{a}}$ & $307,8 \pm 31,8^{\mathrm{abc}}$ & $394,3 \pm 9,8^{\mathrm{abcd}}$ & $436,0 \pm 15,8^{\text {abcde }}$ \\
\hline $9,0-9,9$ & $200,0 \pm 0,0$ & $230,0 \pm 10,4^{a}$ & $288,6 \pm 24,5^{\mathrm{ab}}$ & $416,0 \pm 27,9^{a b c}$ & $516,6 \pm 18,7^{\mathrm{abccd}}$ & $570,0 \pm 18,5^{\text {abcde }}$ \\
\hline $10,0-10,9$ & $246,7 \pm 10,3$ & $284,4 \pm 8,8^{a}$ & $346,7 \pm 19,5^{\mathrm{ab}}$ & $391,4 \pm 10,3^{\mathrm{abc}}$ & $468,0 \pm 36,8^{\mathrm{abcd}}$ & $635,6 \pm 65,4^{\text {abcde }}$ \\
\hline $11,0-11,9$ & $326,7 \pm 10,3$ & $364,4 \pm 8,8^{\mathrm{a}}$ & $427,8 \pm 35^{\mathrm{ab}}$ & $522,9 \pm 17,3^{\mathrm{abc}}$ & $640,0 \pm 66,5^{\mathrm{abcd}}$ & $865,0 \pm 113,5^{\text {abcde }}$ \\
\hline
\end{tabular}

$a, b, c, d, e(p<0,05)$. ${ }^{a}$ Diferencia significativa en relación a la categoría de deficiente de la misma edad, bdiferencia significativa en relación a la categoría de malo de la misma edad, 'diferencia significativa en relación a la categoría de regular de la misma edad, ddiferencia significativa en relación a la categoría de bueno de la misma edad, ediferencia significativa en relación a la categoría de muy bueno de la misma edad. 


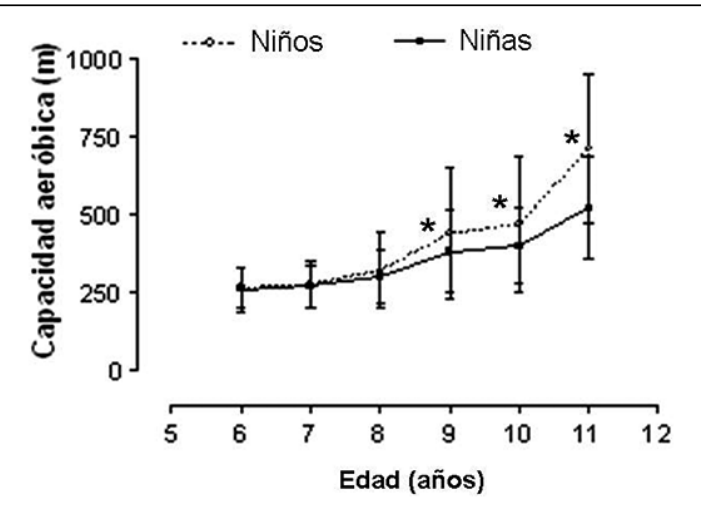

Figura 1. Capacidad Cardio-respiratoria $(m)$ de niños escolares en función de la edad y sexo. $*(p<0,05)$ diferencia significativa por edad en relación a los niños.

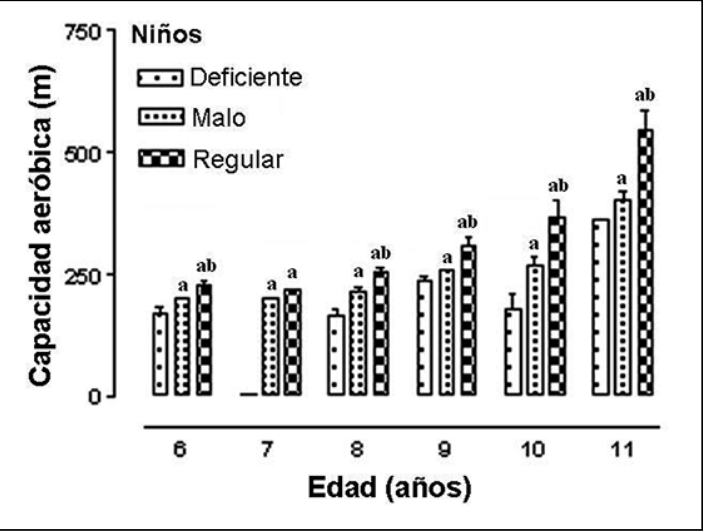

Figura 2. Comparación entre los metros recorrido (capacidad cardio-respiratoria) por edad a partir de tres categorías ${ }^{a, b}$ : $(p<0,05)$, ${ }^{a}$ Diferencias significativa en relación a la categoría deficiente. ${ }^{b}$ Diferencias significativa en relación a la categoría malo.

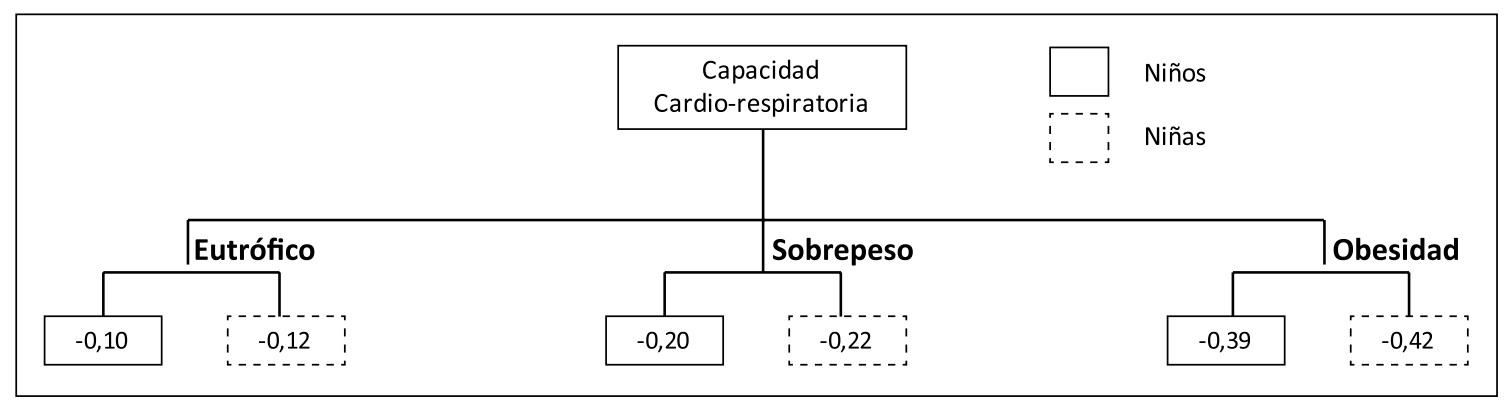

Figura 3. Correlación de Pearson entre el estado nutricional y la capacidad cardio-respiratoria en niños y niñas.

Tabla 3. Niveles de capacidad cardio-respiratoria de escolares de ambos sexos

\begin{tabular}{|c|c|c|c|c|c|c|c|c|c|c|c|c|}
\hline \multirow{2}{*}{$\begin{array}{l}\text { Edad en } \\
\text { años, rango }\end{array}$} & \multicolumn{2}{|c|}{ Deficiente } & \multicolumn{2}{|c|}{ Malo } & \multicolumn{2}{|c|}{ Aceptable } & \multicolumn{2}{|c|}{ Bueno } & \multicolumn{2}{|c|}{ Muy bueno } & \multicolumn{2}{|c|}{ Excelente } \\
\hline & $f$ & $\%$ & $\mathbf{f}$ & $\%$ & $f$ & $\%$ & $f$ & $\%$ & $f$ & $\%$ & $f$ & $\%$ \\
\hline \multicolumn{13}{|l|}{ Niños } \\
\hline $6,0-7,9$ & 4 & 7,1 & 8 & 14,3 & 14 & 25 & 12 & 21,4 & 9 & 16,1 & 9 & 16,1 \\
\hline $7,0-7,9$ & 0 & 0,0 & 7 & 10,4 & 11 & 16,4 & 32 & 47,8 & 10 & 14,9 & 7 & 10,4 \\
\hline $8,0-8,9$ & 6 & 8,8 & 10 & 14,7 & 15 & 22,1 & 15 & 22,1 & 15 & 22,1 & 7 & 10,3 \\
\hline $9,0-9,9$ & 7 & 9,6 & 10 & 13,7 & 15 & 20,5 & 19 & 26,0 & 14 & 19,2 & 8 & 11,0 \\
\hline $10,0-10,9$ & 6 & 10,2 & 8 & 13,6 & 13 & 22 & 16 & 27,1 & 10 & 16,9 & 6 & 10,2 \\
\hline $11,0-11,9$ & 6 & 8,5 & 11 & 15,5 & 14 & 19,7 & 16 & 22,5 & 11 & 15,5 & 13 & 18,3 \\
\hline Total & 29 & 7,36 & 54 & 13,71 & 82 & 20,81 & 110 & 27,92 & 69 & 17,51 & 50 & 12,69 \\
\hline \multicolumn{13}{|l|}{ Niñas } \\
\hline $6,0-7,9$ & 4 & 5,0 & 6 & 7,5 & 24 & 30 & 20 & 25,0 & 13 & 16,3 & 13 & 16,3 \\
\hline $7,0-7,9$ & 6 & 9,8 & 6 & 9,8 & 16 & 26,2 & 9 & 14,8 & 14 & 23 & 10 & 16,4 \\
\hline $8,0-8,9$ & 4 & 6,3 & 6 & 9,5 & 13 & 20,6 & 23 & 36,5 & 7 & 11,1 & 10 & 15,9 \\
\hline $9,0-9,9$ & 2 & 3,4 & 12 & 20,7 & 14 & 24,1 & 10 & 17,2 & 12 & 20,7 & 8 & 13,8 \\
\hline $10,0-10,9$ & 6 & 9,5 & 9 & 14,3 & 15 & 23,8 & 14 & 22,2 & 10 & 15,9 & 9 & 14,3 \\
\hline $11,0-11,9$ & 6 & 7,9 & 9 & 11,8 & 23 & 30,3 & 14 & 18,4 & 16 & 21,1 & 8 & 10,5 \\
\hline Total & 28 & 6,98 & 48 & 11,97 & 105 & 26,18 & 90 & 22,44 & 72 & 17,96 & 58 & 14,46 \\
\hline
\end{tabular}

Diferencia significativa en ambos géneros. Niños: $\chi^{2}=39,0(p=0,0371)$ y niñas: $\chi^{2}=38,1(p=0,0450)$. 


\section{Discusión}

Conocer el nivel de capacidad cardiorespiratoria en los niños de forma individualizada es importante para identificar el riesgo cardiovascular futuro de una persona ${ }^{14-16}$. Para la valoración de esta capacidad, básicamente se utiliza la medida del consumo máximo de oxígeno $\left(\mathrm{VO}_{2} \max \right)^{17,18}$, de esta forma, cuanto mayor es el valor de $\mathrm{VO}_{2}$ max medido de un individuo, mayor será la cantidad y la intensidad de trabajo aeróbico que es capaz de realizar, adquiriendo en consecuencia un mejor rendimiento en la actividad física ${ }^{19,20}$.

La estimación del consumo máximo de oxígeno en niños y adolescentes están aún en fase de ajuste ${ }^{21}$, debido a la inmadurez del metabolismo aeróbico. En este sentido, se sugiere estimar sus valores a través de otras herramientas como la alometria ${ }^{22}$, o a través de metros recorridos, lo que posibilitaría una mejor explicación de los resultados alcanzados. Por ello, la valoración e interpretación del $\mathrm{VO}_{2}$ max en jóvenes aún se encuentra en fase de controversia ${ }^{18}$, dado que no todos los niños y adolescentes crecen y maduran a la misma velocidad y ritmo.

Desde esa perspectiva, los resultados del presente estudio fueron analizados a partir de la distancia recorrida, lo que permitió diagnosticar la capacidad cardio-respiratoria de los niños de ambos sexos. De hecho, en el estudio analizando en función de la edad cronológica los valores promedios aumentan de forma lineal en ambos sexos, observándose mayor capacidad en los niños a partir de los 9 hasta los 11 años, en concordancia con la literatura ${ }^{18,23}$, aunque algunos estudios ${ }^{24,25}$ sugieren la aplicación de escalas alométricas para descartar la influencia del peso corporal. En el presente estudio no se consideró el ajuste alométrico, lo que de alguna forma podría limitar los resultados, una vez que serían favorecidos los niños delgados en relación a los más pesados.

Observamos también que existe diferencias entre las categorías de deficiente, malo y aceptable en ambos géneros y en todas las edades, donde se determinó que $19 \%$ de los niños y $21 \%$ de las niñas se encuentran por debajo de los valores de la capacidad cardio-respiratoria considerado como aceptable, lo que muestra bajos niveles de capacidad cardio-respiratoria durante la infancia. Adicionalmente cuando se correlacionó las categorías del estado nutricional con la capacidad cardio-respiratoria, el coeficiente de Pearson mostró valores negativos débiles para los niños con sobrepeso $(\mathrm{r}=-0,20$ a $-0,22$ ) y moderados para los niños con obesi$\mathrm{dad}(\mathrm{r}=-0,39 \mathrm{a}-0,42)$. Esto demuestra que una baja capacidad cardio-respiratoria se relaciona con el exceso de peso corporal, por lo que la capacidad aeróbica desempeña un papel protector sobre el riesgo cardiovascular en niños ${ }^{26}$ y adolescentes ${ }^{27}$.

Por lo tanto, en este estudio 1 de cada 5 niños se encuentra con posibilidades de presentar algún evento de índole cardiovascular, dado que, para llegar a tener una capacidad cardiorespiratoria aceptable existe una diferencia entre $25-50 \%$ y de $24-35 \%$ entre la categoría de deficiente a aceptable, y de un $9-28 \%$ y $10-$ $22 \%$ entre la categoría de malo a aceptable en niños de ambos sexos, respectivamente. Esta es una alta frecuencia que requiere atención específica por parte de las autoridades políticas, sanitarias y educativas, los que son considerados ejes esenciales para una detección precoz y una actuación inmediata ${ }^{28}$ en casos como los observados en el presente estudio. No podemos afirmar categóricamente que los bajos niveles de capacidad cardiorespiratoria pueden repercutir sobre la salud cardiovascular de los niños estudiados, dado que el método utilizado para valorar dicha capacidad fue por medio de una prueba indirecta de campo, aunque dicho procedimiento no invalida los resultados, en vista de que tales pruebas son aplicadas y utilizadas cotidianamente en la escuela y podría ser considerada como una herramienta válida y confiable para la valoración del riesgo cardiovascular de niños escolares.

Es conocido que la inactividad física y el estilo de vida cada vez más sedentario se ha incrementado en altos índices de prevalencia y obesidad en todo el mundo ${ }^{29}$, extendiéndose con rapidez entre los niños de edad escolar, sobre todo, en lugares donde se ha reducido la recreación y los programas de actividad física, como se describe en un estudio en niños mexicanos, donde Hernández et $\mathrm{al}^{30}$ evidenciaron 
una alta prevalencia de obesidad (20\%), inclusive, algunos estudios nacionales realizados a nivel del mar ${ }^{31}$ muestran $22 \%$ de prevalencia de sobrepeso y $7 \%$ de obesidad, así como en escolares de moderada altitud ${ }^{32}$ la prevalencia de sobrepeso fue del $27 \%$ en niños y del $21 \%$ en niñas y de obesidad $14,5 \%$ en niños y de $6 \%$ en niñas, respectivamente.

Estas evidencias hacen suponer que el tipo de ejercicio, el tiempo, la intensidad de las actividades físicas desarrolladas por los escolares y la frecuencia (una vez por semana) no son suficientes para alcanzar una capacidad cardio-respiratoria adecuada como indicador de salud. Pues de hecho, un bajo nivel de capacidad física es considerado como fuerte predictor de enfermedades cardiovasculares, no sólo en sujetos con sobrepeso u obesidad, sino también en sujetos eutróficos ${ }^{33}$. Por lo tanto, los jóvenes que presentan baja condición física y altos niveles de sobrepeso y obesidad muestran un aumento de riesgo en enfermedades metabólicas ${ }^{32}$.

Destacamos también que durante el estudio no se pudo controlar variables como el tipo de alimentación, perfil lipídico y la presión arterial de los niños estudiados, lo que hubiera permitido interpretar de mejor forma los resultados obtenidos. Sugerimos en futuros estudios considerar el control de tales variables, así como la posibilidad de comparar con un grupo de escolares que realicen actividad física entre tres a cuatro veces por semana, lo que evidentemente podría contribuir en determinar los beneficios de la actividad física sobre la capacidad cardio-respiratoria.

Se concluye que el $19 \%$ de los niños y $21 \%$ de las niñas escolares que viven a moderada altitud se encuentran con bajos niveles de capacidad cardio-respiratoria, además se evidenció que una baja capacidad cardio-respiratoria se relaciona con el exceso de peso corporal, sugiriendo que la capacidad aeróbica podría desempeñar un papel protector sobre el riesgo cardiovascular a edades tempranas. En este sentido, el Ministerio de Educación del Perú, a través de las directrices curriculares debería considerar la posibilidad de incrementar el número de horas y sesiones en los programas de actividad física de los centros escolares de moderada altitud, con la intención de fomentar la práctica regular del ejercicio físico, los deportes, la danza y la recreación.

\section{Referencias}

1.- Leiva M, Casajús JA: Cineantropometría. Condición física. Estilo de vida de los escolares aragoneses (7 a 12 años). Zaragoza: Diputación General de Aragón; 2004.

2.- Caspersen CJ, Powell KE, Christenson GM: Physicalactivity, exercise, and physical-fitness-Definitions and distinctions for Health-Related Research. Public Health Reports 1985; 100 (2): 126-31.

3.- Guedes DP, Guedes JERP: Controle do Peso Corporal: composición corporal, atividade física e nutrição. Río de Janeiro: Shape, 2003.

4.- American Collage of Sports Medicine. Manuel para teste de esforco e prescripcao de exercicio. 4. ed. Rio de Janeiro: Revinter Ltda. 1996.

5.- Astrand PO: Factores que se han de medir. In: Astrand $\mathrm{R}$, Shephard RJ (Eds). La resistencia en el deporte. pp, 201-203, 2 ed. Barcelona, Editorial Paidotribo, 2000.

6.- James PT: Obesity: the worldwide epidemic. Clin Dermatol 2004; 22: 276-80.

7.- World Health Organization. Obesity. Preventing and managing the global epidemic. Report of a WHO consultation of obesity. Geneva: WHO: 1998.

8.- Ross WD, Marfell-Jones MJ: Kinanthropometry. In: J.D. MacDougall, H.A, Wenger, y H.J, Geen (Eds). Physiological testing of elite athlete. pp, 223-308, London, Human Kinetics 1991.

9.- Boileau RA, Lohman TG, Slaughter MH: Exercise and body composition in children and youth. Scan, J. Sports Sci 1985; 7, 17-27.

10.- Hernández-Rodrigues M: Alimentación infantil. Madrid: Díaz de Santos, 1993: 11-23.

11.- De Onis M, Garza C, Onyango AW, Borghi E: Comparisson of the WHO child growth standards and CDC 2000 Growth charts. J Nutr 2007; 137 (1): 144-8.

12.- Leger LA, Mercier D, Gadoury C, Lambert J: The multistage 20-m run test for aerobic fitness. J. Sport Sci 1988; (6): 93-101.

13.- Cossio-Bolaños MA, Arruda M: Propuesta de valores normativos para la evaluación de la aptitud física en niños de 6 a 12 años de Arequipa, Perú. Rev Med Hered 2009; 20: 206-12.

14.- Janz KF, Dawson JD, Mahoney LT: Increases in physical fitness during childhood improve cardiovascular health during adolescence: the Muscatine Study. Int J Sports Med 2002; 23 Suppl 1: 15-21. 
15.- Boreham C, Twisk J, Murray L, Savage M, Strain JJ, Cran $G$ : Fitness, fatness, and coronary heart disease risk in adolescents: the Northern Ireland Young Hearts Project. Med Sci Sports Exerc 2001; 33: 270-4.

16.- Boreham C, Twisk J, Neville C, Savage M, Murray L, Gallagher A: Associations between physical fitness and activity patterns during adolescence and cardiovascular risk factors in young adulthood: The Northern Ireland Young Hearts Project. Int J Sports Med 2002; 23 Suppl 1: 22-6.

17.- McArdle W, Katch F, Katch V: Fisiologia do exercício: energia, nutrição e desempenho humano. 3. ed. Rio de Janeiro: Guanabara Koogan 2002: 178-201.

18.- Armstrong $N$ : Aerobic fitness of children and adolescent. J Pediatr (Rio J) 2006; 82: 406-8.

19.- Kiss MA: Esporte e exercício: avaliação e prescrição. São Paulo: Roca, 2003. p. 63-91.

20.- Tritschler $K A$ : Medida e avaliação em educação física e esportes de Barrow \& McGee. 5. ed. Barueri: Manole, 2003: 273-305.

21.- Barbosa-Montoro S, Teixeira-Mendes R, Arruda M, Bicudo-Zeferino MA: Aptidão Aeróbica de crianças e adolescentes obesos: Procedimientos de controle. Revista Brasileira de Ciencas da Saude 2009. año VII. N.19.

22.- Moncada-Jiménez J, Lachowetz A: Escalas Alométricas: Un justo criterio en el Proceso de Evaluación en las Ciencias del Movimiento Humano. Red de Revistas Científicas de América Latina y El Caribe, España y Portugal 2001; 5 (1): 95-102.

23.- Malina RM, Bouchard C, Bar-Or O: Growth, maturation and physical activity. Champaign: Human Kinetics, 2004.

24.- Welsman JR, Armstrong N, Nevill AM, Winter EM, Kirby $B J$ : Scaling peak $\mathrm{VO}_{2}$ for differences in body size. Med sci sports Exerc 1996; 28: 259-65.

25.- Armstrong N, Welsman J: Peak oxygen uptake in rela- tion to growth and maturation. Eur J Appl Physiol 2001; 85: 546-51.

26.- Raitakari OT, Taimela S, Porkka KV, et al: Associations between physical activity and risk factors for coronary heart disease: the Cardiovascular Risk in Young Finns Study. Medicine and Science in Sports and Exercise 1997; 29, 1055-61.

27.- García-Arteroa E, Ortega F, Ruiza J, et al: El perfil lipídico-metabólico en los adolescentes está más influido por la condición física que por la actividad física (estudio AVENA). Rev Esp Cardiol 2007; 60 (6): 581-8.

28.- Ortega F, Ruiz J, Castillo M, et al: Bajo nivel de forma física en los adolescentes españoles. Importancia para la salud cardiovascular futura (Estudio AVENA). Rev Esp Cardiol 2005; 58 (8): 898-909.

29.- Peña $M$, Bacallao J: La obesidad en la pobreza: un nuevo reto para la salud pública. Washington DC: Organización Panamericana de la Salud; 2000.

30.- Hernández B, Cuevas-Nasu L, Shamah-Levy T, et al: Factores asociados con sobrepeso y obesidad en niños mexicanos de edad escolar: resultados de la Encuesta Nacional de Nutrición, 1999. Salud Pública Mex 2003; 45 (supl 4): 551-57.

31.- Bustamante VA, Seabra AF, Garganta RM, et al: Efectos de la actividad física y del nivel socioeconómico en el sobrepeso y obesidad de escolares, Lima Este 2005. Rev Perú Med exp salud pública 2007; 24 (2): 121-8.

32.- Cossio-Bolaños MA, Eduardo-Hespanhol J, Merma Monje C, Arruda M: Desempeño de la condición física relacionada a la salud en función del índice nutricional en niños de moderada altitud. Pediatría de México 2011. 13(3).

33.- Wei M, Kampert JB, Barlow CE, et al: Relationship between low cardiorespiratory fitness and mortality in normal-weight, overweight, and obese men. JAMA 1999; 282: 1547-53. 